\title{
Alterations of Thyroid Hormones and Interleukin-6 (IL-6) Level in End Stage Renal Disease.
}

\author{
Abdel-Aziz. F. Abdel-Aziz ${ }^{1}$, Ibrahim. A. Abdel-Aal' ${ }^{2}$ Mohamed. Sh. Zormba ${ }^{3}$, \\ Nashwa. A. Mohamed. ${ }^{4}$ \\ ${ }^{1}$ (Department of Chemistry, Faculty of Science/ Mansoura University, Mansoura, Egypt) \\ ${ }^{2}$ (Department of Clinical Pathology, Faculty of Medicine/ Mansoura University, Mansoura, Egypt) \\ ${ }^{3}$ (Department of Chemistry, Faculty of Science/ Port Said University, Port Said, Egypt) \\ ${ }^{4}$ (Department of Chemistry, Faculty of Sciencel Port Said University, Port Said, Egypt)
}

\begin{abstract}
Chronic kidney disease (CKD) causes alterations in thyroid hormones without underlying thyroid disorder this syndrome known as nonthyroidal illness syndrome (NTIs). This syndrome is mainly characterized by decrease in total triiodothyronine (tT3) level with normal thyroidstimulating hormone (TSH) level. The low tT3 level in CKD patients have been correlated with higher levels of inflammation markers such as interleukin -6 (IL-6). This study included 65 hemodialysis (HD) patients and 25 healthy volunteers as normal control group. Serum total triiodothyronine (tT3) and total thyroxin (tT4) levels were low, but serum TSH level was normal when compared with control group. These results associated with high level of IL-6. Also, there were highly significant negative correlation between serum IL-6 and tT3 level but mild significant negative correlation with tT4 level in HD patients. On the other hand, there was no significant correlation between serum IL-6 and TSH level.
\end{abstract}

Keywords: Nonthyroidal illness syndrome, Thyroid- stimulating hormone, Interleukin -6 Hemodialysis, Total Thyroxin.

\section{Introduction}

Chronic renal failure (CRF) is a world-wide serious health and economic issue with an increasing incidence and prevalence. The term chronic renal failure usually means the end stage of chronic kidney disease with a decline in glomerular filtration rate (GFR) below $0.25 \mathrm{~mL} / \mathrm{sec}$. (Zadrazil, 2011). As kidney function declines, there is a progressive deterioration in mineral homeostasis and changes in circulating hormones (Eknoyan , 2004).Hemodaylsis (HD) remains the most common technique for treatment of CRF patients (Yilmaz et al., 2000). HD is associated with alterations in the concentration of thyroid hormones (Zerrati et al., 2011). The kidney normally plays an important role in the metabolism, degradation, and excretion of several thyroid hormones. Therefore, the impairment in kidney function may lead to disturbed thyroid physiology (Sarookhani, 2007).

In this respect, thyroid hormones are essential for an adequate growth and development of the kidney (Avasthi et al., 2001). Conversely, the kidney is not only an organ for metabolism and elimination of Thyroid hormone, but also a target organ of some of the iodothyronines' actions (Iglesias and Díez, 2009). It is possible in the individual CRF patients to assess thyroid status accurately by physical diagnosis and thyroid function testing (Palmer et al., 2011).

Although the pathogenesis of hypothalamic-pituitary-thyroid axis abnormalities encountered in nonthyroidal illness (NTI) as CRF remains elusive, it is currently agreed that it may be related to increased cytokines production (Abozenah et al., 2008).

Cytokines are small cell-signaling protein molecules that are secreted by numerous cells and are a category of signaling molecules used extensively in intercellular communication. Cytokines act as key communicators for immune cells and maintaining a delicate balance in the level of these communicators is vital for health - in many chronic diseases, this balance is disrupted (Hafler, 2007). Interleukin-6 (IL-6) is a pleiotropic cytokine. IL-6 has been implicated in the generation and propagation of chronic inflammation. Initially in acute inflammation, proinflammatory cytokines promote neutrophil accumulation and the release of IL-6 (Barnes et al., 2011).

In this study, we assess pituitary thyroid axis function in patients with chronic renal failure and its relation with serum Interleukin- 6 concentration, in order to: estimate the potential use of thyroid hormones testing as morbidity indicator in patients with chronic renal failure and illustrate the potential link between thyroid function and Interleukin-6. 


\section{II.1 Subjects}

\section{Subjects and Methods}

This study included 90 subjects; 65 HD patients, their age range from 23 to 68 year with mean age $40.17 \pm 12.58$ year and with the average duration of dialysis $2.21 \pm 0.12$ years, they were $47(72.3 \%)$ males and $18(27.7 \%)$ females, all patients were undergoing dialysis treatment following diagnosis of end stage renal disease (ESRD) by nephrologists in the Urology and Nephrology Center, Mansoura University, hemodialysis unit, each patient was dialyzed 3 times per week also, 25 healthy volunteers as normal control group their age range from 25 to 70 year with mean age $42.35 \pm 14.03$, they were $15(60 \%)$ male and $10(40 \%)$ female.The control subjects were collected from donner blood bank,Mansoura University. Serum creatinine, uric acid, blood urea nitrogen (BUN) and liver function parameters as, serum albumin ,total bilirubine,liver enzymes (SGPT, SGOT and ALP ) were tested. The control subjects had normal kidney functions, normal thyroid functions . They were free from any kidney diseases. The studied subjects(patients and controls) were in the same socioeconomic class and had similar nutritional habits.

\section{II.2 Blood sample collection and examination}

Five milliliters venous blood were collected using a disposable plastic syringe from each patient and healthy individuals.All samples of patients were collected before the dialysis ( pre- hemodialysis) One milliliter from $5 \mathrm{ml}$ was collected on EDTA for evaluation of hemoglobin level. The rest of blood $(4 \mathrm{ml})$ were collected on free tubes without addition anticoaggulants, were centrifuged at $3000 \mathrm{rpm}$ for 5 minutes to obtain serum for estimation of interleukin-6 (IL-6) thyroid hormones (T3,T4 and TSH), kidney functions testes (creatinine ,BUN and uric acid ) and liver functions testes in ESRD patients and control subjects. Interleukin-6 (IL-6) was estimated by: Human IL-6 ELISA Kit, Boster Immunoleader, Boster Biological Technology Co., Inc 40459 Encyclopedia Circle, Fremont, CA 94538 (Kiecolt-Glaser et al ., 2003) using Teco Diagnostics 98 Elisa Strip Reader, CA 92807,USA . Total Triiodothyronine (tT3) and Total Thyroxine (tT4) levels were estimated by: Enzyme Immuno assay Test Kit , Monobind Inc., Lack Forest, CA 92630,USA (Braverman, 1996) using the same apparatus ( Teco Diagnostics 98 Elisa Strip Reader, CA 92807, USA). Thyroid stimulating hormone (TSH) level was determined by: Immuno flurescent Immuno assay (Windosford et al., 1996) using (Mini Vidas Biomerieux CA 69280,France).

\section{II.3 Statistical analysis}

Data were obtained using Statistical package for social Sciences (SPSS) version 19.0 software. Data were expressed as means \pm standard deviation (SD) .Results of ESRD patients and control subjects were performed using chi- square analysis, independent t-test . Correlation between parameters was determined by pearson 's correlation coefficient coefficient (r). Chi square and odds ratio were calculated with $95 \%$ confidence interval .A p- value less than 0.05 was considered statistically significant.

\section{Results}

As observed in table (1), there were no significant difference of age and body mass index (BMI) in ESRD patients when compared with control subjects. The systolic blood pressure (SBP) and diastolic blood pressure (DBP) were significantly higher in ESRD patients than in control subjects, $(p<0.001)$. On the other hand, level of hemoglobin was significantly lower in ESRD patients as compared to control subjects $(\mathrm{P}<0.001)$.

\section{III.1 Demographic data}

The demographic data of HD patients and control group are presented in Table (1).

Table 1 Demographic and clinical data of studied subjects.

*Highly

\begin{tabular}{|c|c|c|c|}
\hline parameters & $\begin{array}{c}\text { ESRD Patients (n=65) } \\
\text { M } \pm \text { SD }\end{array}$ & $\begin{array}{c}\text { Control subjects }(\mathbf{n}=\mathbf{2 5}) \\
\text { M } \pm \text { SD }\end{array}$ & p- value \\
\hline Age (years) & $40.17 \pm 12.58$ & $42.35 \pm 14.03$ & NS \\
\hline BMI $(\mathrm{kg} / \mathrm{cm} 2)$ & $22.82 \pm 4.73$ & $23.83 \pm 3.88$ & NS \\
\hline SBP $(\mathrm{mmHg})$ & $144.61 \pm 10.52$ & $118.23 \pm 2.31$ & $<0.001^{*}$ \\
\hline DBP $(\mathrm{mmHg})$ & $92.01 \pm 3.21$ & $72.83 \pm 4.05$ & $<0.001^{*}$ \\
\hline Hemoglobin(g/dL) & $8.64 \pm 1.01$ & $12.15 \pm 0.92$ & $<0.001^{*}$ \\
\hline Dialysis period/yr & $2.21 \pm 0.12$ & 0 & \\
\hline
\end{tabular}

significant value $(\mathrm{p}<0.05)$. Data were expressed as $($ mean $\pm \mathrm{SD})$. SD: standard deviation. Data were obtained using independent t-test. Body mass index (BMI) .Systolic blood pressure (SBP). Diastolic blood pressure (DBP). 


\section{III.2 Laboratory data}

In The present study, clinical parameters such as creatinine ,blood urea nitrogen (BUN) and uric acid were tested for end stage renal disease (ESRD) patients, $(n=65)$, as well as control subjects $(n=25)$.Table (2) shows the levels of creatinine, BUN and uric acid were significantly increased in ESRD patients when compared with control subjects $(\mathrm{p}<0.001)$.

Table 2 Biochemical Features of studied subjects with control.

\begin{tabular}{|c|c|c|c|}
\hline \multirow{2}{*}{ Variables } & Subject $(n=65)$ & Control $(n=25)$ & \multirow{2}{*}{ P-value } \\
\hline & $\mathbf{M} \pm \mathbf{S D}$ & $\mathbf{M} \pm \mathbf{S D}$ & \\
\hline Creatinine $(\mathrm{mg} / \mathrm{dL})$ & $9.81 \pm 0.33$ & $0.68 \pm 0.14$ & $<0.001 *$ \\
\hline $\mathrm{BUN}(\mathrm{mg} / \mathrm{dL})$ & $88.66 \pm 8.22$ & $7.21 \pm 0.52$ & $<0.001 *$ \\
\hline Uric Acid( mg/dL) & $8.11 \pm 0.22$ & $4.33 \pm 0.31$ & $<0.001 *$ \\
\hline Albumin $(\mathrm{g} / \mathrm{dL})$ & $2.82 \pm 0.18$ & $4.55 \pm 0.14$ & $<0.001 *$ \\
\hline $\mathrm{TP}(\mathrm{g} / \mathrm{dL})$ & $6.11 \pm 0.21$ & $7.88 \pm 0.33$ & $<0.001 *$ \\
\hline ALP(IU/I) & $125.14 \pm 12.17$ & $80 \pm 4.41$ & $<0.001 *$ \\
\hline $\mathrm{TB}(\mathrm{mg} / \mathrm{dL})$ & $0.75 \pm 0.08$ & $0.71 \pm 0.04$ & NS \\
\hline $\mathrm{ALT}(\mathrm{U} / \mathrm{I})$ & $30.24 \pm 0.54$ & $27.13 \pm 0.77$ & NS \\
\hline $\operatorname{AST}(\mathrm{U} / \mathrm{I})$ & $32.16 \pm 0.35$ & $30.23 \pm 0.44$ & NS \\
\hline Sodium(mmol/L) & $125.19 \pm 3.15$ & $144.12 \pm 2.21$ & $<0.001^{*}$ \\
\hline Potassium(mmol/L) & $5.43 \pm 0.22$ & $4.12 \pm 0.11$ & $<0.001 *$ \\
\hline Calcium ( mg/dL) & $7.88 \pm 0.66$ & $10.18 \pm 0.11$ & $<0.001^{*}$ \\
\hline Phosphorus (mg/dL) & $5.51 \pm 0.17$ & $3.21 \pm 0.08$ & $<0.001^{*}$ \\
\hline IL-6 (ng/L) & $35.53 \pm 9.27$ & $3.48 \pm 0.62$ & $<0.001^{*}$ \\
\hline $\mathrm{tT} 3(\mathrm{mg} / \mathrm{dL})$ & $0.81 \pm 0.15$ & $1.04 \pm 0.26$ & $<0.001 *$ \\
\hline tT4 ( Ug/dL ) & $7.21 \pm 1.12$ & $8.01 \pm 0.84$ & 0.01 \\
\hline TSH (UIU/mL) & $2.26 \pm 0.46$ & $2.33 \pm 0.62$ & NS \\
\hline
\end{tabular}

In table (2) biochemical parameters such as albumin, total protein (TP),alkaline phosphatase (ALP) enzyme ,total bilirubin (TB), alanine aminotransferase (ALT) enzyme, asparate aminotransferase (AST) enzyme, sodium, potassium, calcium, phosphorus, interleukin-6 (IL-6),total triiodothyronine (tT3) hormone, total thyroxine (tT4) hormone and thyroid -stimulating hormone (TSH) were tested for end stage renal disease (ESRD) patients, $(\mathrm{n}=65)$, as well as control subjects $(\mathrm{n}=25)$. The levels of albumin, TP, sodium, calcium, tT3 $(\mathrm{p}<0.001)$ and tT4 $(\mathrm{p}=0.01)$ were low significant in ESRD patients when compared with control subjects, but the levels of ALP enzyme, potassium ,phosphorus and IL-6 were higher significant in ESRD patints than in control subjects.

On the other hand, the levels of ALT,AST enzymes, total bilirubin and TSH levels of ESRD were not significant compared with control subjects .

\section{III.3 Analysis of correlation}

As shown in table (3), concentration of serum interleukin-6 (IL-6) in ESRD patients revealed high significant negative correlation $(\mathrm{p}<0.05)$ with total triiodothyronine hormone (tT3 ) $(\mathrm{r}=-0.767)$. Also, there was mild significant negative correlation $(p<0.05)$ between IL -6 and total thyroxine hormone (tT4) in ESRD patients $(\mathrm{r}=-0.318)$ Figures (1 and 2). The results showed that there was no significant correlation between interleukin -6 (IL-6) and thyroid stimulating hormone (TSH) in End Stage Renal Disease( ESRD) patients, $\mathrm{n}=65$.

Table 3 Pearson's Correlation between Interleukin-6 ( IL-6) Level and Thyroid hormones in ESRD patients. $\mathrm{p}>0.05$; not significant (NS), $\mathrm{p}<0.05$; Significant, $\mathrm{p}<0.001$; highly significant

\begin{tabular}{|c|c|c|}
\hline \multicolumn{3}{|c|}{ IL-6 (ng/L) (n=65) } \\
\hline Parameters & r-value & p-value \\
\hline tT3 $(\mathrm{mg} / \mathrm{dL})$ & -0.767 & $<0.001^{*}$ \\
\hline $\mathrm{tT} 4(\mathrm{Ug} / \mathrm{dL})$ & -0.318 & 0.018 \\
\hline $\mathrm{TSH}(\mathrm{UIU} / \mathrm{mL})$ & -0.130 & 0.344 \\
\hline
\end{tabular}



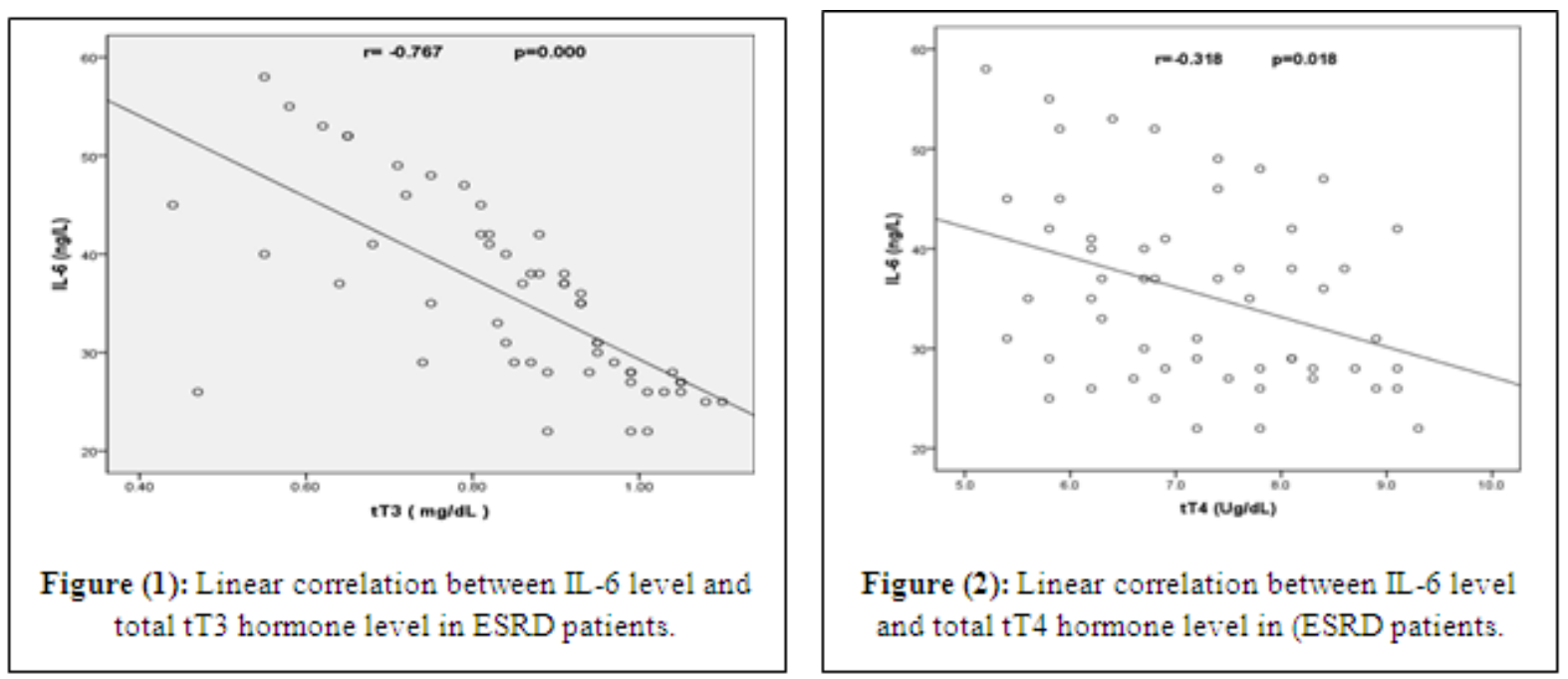

\section{Discussion}

Chronic kidney disease (CKD) is well -known cause of nonthyroidal iilness syndrom (NTIs) (i.e., altreation in thyroid hormones without underlying thyroid disorder) and affects all levels of the hypothalamus pituitary thyroid axis. The interpretion of thyroid functions in patients with CKD is even more complicated by the declination in glomerular filtration rate (GFR) (Chopra, 1996 and Tarng, 2012). The interactions between kidney and thyroid functions are known for many years (Basu and Mohaptra , 2012). CKD affects thyroid function in multiple ways, including low levels of circulating thyroid hormone concentration, altered peripheral hormone metabolism, disturbed binding to carrier proteins , possible reduction in tissue of thyroid hormone content and increased iodine stores in the thyroid gland. Both serum triiodothyronine (T3) and total thyroxine (T4) levels are reduced (Alsaran et al ., 2011). All the levels of hypothalamic- pituitary-thyroid axis involved alterations in hormone production and excretion, this was observed in pre-dialysis patients with CKD that had an increased risk of hypothyroidism (Lo et al ., 2005).

Serum T3 levels were consistently found to be low ,serum T4 concentrations have been reported as low, normal or high( Avasthi et al ., 2001). The most common thyroid function abnormality in CKD is low T3 level (Kaptein , 1996 and Wiederkehr, 2004 ). Low T3 levels occurs in CKD due to several reasons .Fasting ,chronic metabolic acidosis and chronic protein malnutrition affect iodothyronine deiodation ,as well as protein binding of T3, reducing the peripheral convertion of T4 to T3 (Zoccali et al ., 2005). Decreased T3 levels are not due to increased T3 degradation or to reduced thyroidal T3 secretion but is a result of impaired extrathyroidal T4 toT3 convertion, while thyroid gland production of T3 is normal (Singh et al ., 2006). The reduction in $\mathrm{T} 4$ is attributed to the presence of circulating inhibtors, which impair binding of $\mathrm{T} 4$ to thyroxinbinding globulin. Despite decreased circulating T4 and T3 levels ,thyroid- stimulating hormone (TSH) levels were not elevated . This absence of elevated TSH levels is not due to dysfunction of the hypothalmo-pituitary axis, this confirmed that these patients were NTI (Alsaran et al ., 2011).

Many studies have focused on the role of inflammatory cytokines which are known to be important mediators of CKD (Davies et al., 1996). The concentration of several cytokines are elevated in patients with CKD due to their decreased renal clearance (Schindler, 2004). Several studies have shown that proinflammatory cytokines may inhibit T3 production (Ozawa et al ., 1988 and Boelen et al ., 1993)The low T3 levels especially total T3 not free T3 in CKD patients have been correlated with higher levels of inflammation markers such as . Intreleukine-6 (IL-6) (Zoccali et al., 2005 and Carrero et al., 2007) IL-6 is the major mediator of the acute phase response, elevated in CKD patients (Filho et al ., 2012). Elevated proinflammatory cytokine (IL-6) was estimated in NTI patients as in CKD (Filiopoulos and Vlassopoulos, 2009). The present study revealed that there were low significant diference in the levels of serum triiodothyronine (T3) and thyroxin (T4) between CKD patients and controls. On the other hand, there was no significant in thyroid stimulating hormone (TSH) as compared with control group.These results were in accordance with data reported by (Horimoto and Nishikawa, 1988;Xess et al ., 1999 and Abo- zenah et al , 2008), but in contrast to the result of the study taken by (Kayima et al.,1992). These results may explain some features of hypothyroidism and thyroid hormone resistance observed in chronic renal failure (CRF) patients (Santos et al ., 2005) . 
Previous reports were indicated a significant decrease in the level of serum triiodothyronine (T3)with normal level of thyroid stimulating hormone(TSH)as compared with healthy controls (song et al ., 2009) these were in aggrement with the presenet results.

In this study, we observed elevated levels of serum IL-6 when compared with control group as table (2). A highly significant inverse correlation between IL-6 and T3 levels was estimated as in figuer (1), this in accordance with (Lo et al .,2005). Also, there was a mild negative correlation between serum IL-6 and total T4 levels as in figure (2), but there was no correlation between serum IL-6 and serum TSH as in table (3). This results were in aggrements with (Davies et al., 1996 and Abozenah et al .,2008).

\section{Conclusion}

Prevalence of hypothyroidism disease in patients with NTIs increases with long-term hemodialysis According to this study, we found that low serum tT3 low serum tT4 levels and high level of IL-6.we suggest that thyroid hormone especially T3 measurements as useful morbidity indicator for CKD.

\section{References}

[1]. Abozenah, H. ; Shoeb, S.; Sabry, A. and Ismail, H. (2008): Relation between thyroid hormone concentration and serum levels of interleukin-6 and interleukin-10 in patients with nonthyroidal illness including chronic kidney disease. Iran J Kidney Dis, 2(1):16-23

[2]. Alsaran, K.; Sabry, A.; Alshahhat, H.; Babgy, E. and Alzaharani, F. (2011): Free Thyroxine, Free Triiodothyronineand Thyroid - Stimulating Hormone before and after dialysis in Saudi patients in end stage renal disease. Saudi.J.Kidney.Dis.Transpl ,22(5):917-921.

[3]. Avasthi, G.; Malhotra, S.; Narang, APS. and Sengupta, S. (2001): Study of thyroid function in patients of chronic renal failure. Indian J Nephrol.; 11: 165-169.

[4]. Barnes, T.C.; Anderson, M.E. and Moots, R.J. (2011): The Many Faces of Interleukin-6. International Journal of Rheumatology,1-6.

[5]. Basu, G. and Mohapatra, A. (2012): Interactions between thyroid disorders and kidney disease. Indian Journal Of Endocrinology and Metabolism.,16(2):204-213.

[6]. Boelen,A.; Platvoet-Ter Schiphorst, M.C. and Wiersinga, W.M. (1993): Association between serum interleukin-6 and serum triiodothyronine in nonthyroidal illness.J.Clin.Endocrinol.Metab,77:1695-1709.

[7]. Braverman, L.E. (1996): Evaluation of thyroid status in patients with thyrotoxicosis. Clin.Chem,42:174-178.

[8]. Carrero, J.J.; Qureshi, A.R.; Axelsson, J.; Yilmaz, M.I.; Rehnmark, S. and Witt, M.R.(2007): Clinical and biochemical implications of low thyroid hormone levels in euthyroid patients with chronic kidney disease. J. Intern.Med,262:690-701.

[9]. Chopra, I.J. (1996): Non thyroidal illness syndrome or euthyroid sick syndrome, Endocr .Pract,2:45-52.

[10]. Davies, P.H.; Black, E.G.;Sheppard, M.C. and Franklyn, J.A.(1996): Relation between serum interleukin-6 and thyroid hormone concentrations in 270 hospital in - patients with non- thyroidal illness.Clinical Endocrinology,44:199-205.

[11]. Eknoyan, G.; Lameire, N. and Barsoum R. (2004): The burden of kidney disease improving global outcomes. Kidney. Int, 66: 1310-1314.

[12]. Filho, R.P.; Lindholm. B.; Axelsson,J. and Stenvinkel, P. (2012): Update on interleukien-6 and its chronic renal failure.Nephrol.Dial.Transplant,8:1043-1045.

[13]. Filiopoulos,V. and Vlassopoulos,D.(2009): Inflmmatory Syndrome in Chronic Kidney Disease: Pathogenesis and Influence on Outcomes.Inflammation. Allergy.Drug Targets, 8:369-382.

[14]. Hafler, ADA. (2007): Cytokines and T cells lymphocytes. Nature Reviews Immununology,423: 15-20.

[15]. Horimoto, M. and Nishikawa,M. (1988): Studies on the pituitary and thyroid function in patients with nonthyroidal illnesses. Nippon Naibunpi Gakkai Zasshi, 64:119-127.

[16]. Iglesias, P. and Díez, J.J. (2009): Thyroid dysfunction and kidney disease. Eur J Endocrinol, 160(4):503-515.

[17]. Kaptein, E.M. (1996): Thyroid hormone metabolism and thyroid disease in chronic renal failure.Endocr.Rev,17:4563.

[18]. Kayima, J.K.;Otleno, L.S.;Gitau,M. and Mwai,S. (1992): Thyroid hormone profiles in patients with chronic renal failure on conservative managements and regular haemodialysis.East.Afr.Med.j,69:333-336.

[19]. Kiecol-Glaser, J.K.; Preacher, K.J.; Maccullum, R.C.; Atkinson, C.; Malarkey. W.B. and Glaser, R. (2003): Chronic stress and age - related increases in the poinflammatory cytokine IL-6. Proc.Nat.Acad.Sci,100: 9090-9095.

[20]. Lo, J.C.; Chertow, G.M.; Go, A.S. and Hsu, C.Y.(2005): Increased Prevalence of subclinical and clinical hypothyroidism in person with chronic kidney disease.Kidney.Int,67:1047-1052.

[21]. Ozwa, M.; Sato, K.; Han, D.C.; Kawakami, M.; Tsushima,T. and Shizume, K.(1988): Effects of tumor necrosis factor-alpha/ cachectin on thyroid hormone metabolism in mice.Endocrinology, 123:1461-1470.

[22]. Palmer, B.F.; Berns, J.S.; Sheridan, A.M. and Henrich, W. (2011): Thyroid function in chronic kidney disease. Uptodate website; http://www.uptodate.com/contents/thyroid-function-in-chronic-kidney-disease .

[23]. Santos, G.M.; Pantoja, C.J.; Costa, E. and Silva. A. (2005): Thyroid hormone receptor binding to DNA and T3dependent transcription activation are inhibited by uremic toxins.Nucl.Recept,4(1): 1 .

[24]. Sarookhani, M.R. (2007): Evaluation of thyroid function test in hemodialysed patients. The journal of Qazvin Univ of Med Science, 10(4): 45-50.

[25]. Schindler, R. (2004): Causes and therapy of microinflammation in renal failure. Nepherol. Dial.Transplant,19:34-40.

[26]. Singh, P.A.; Bobby, Z.; Selvaraj, N. and Vinayagamoorthi, R. (2006): An Evaluation Of Thyroid Hormone Status and Oxidative Stress in Undialyzed Chronic Renal Failure . 
[27]. Song, S.H.; Kwak, I.S.; Lee, D.W.; Kang, Y.H.; Seong, E.Y. and Park, J.S. (2009): The Prevalence of low triiodothyronine according to the stage of chronic kidney disease in subjects with a normal thyroid- stimulating hormone.Nephrol.Dial. Transplant,24:1534-1538.

[28]. Trang, D.C. (2012): Abnormal thyroid function in peritoneal dialysis patients. Journal of the Chinese Medical Association, 75:47-48.

[29]. Wiederkehr, M.R.; Kalogiros, J. and krapf, R. (2004): Correlation of metabolic acidosis improves thyroid and growth hormone axes in haemodialysis patients. Nepherol Dial Transplant,19:1190-1197.

[30]. Wondisford, F.E.;Magner, G.A. and Weinteraub, B.D. (1996): Chemistry and biosynthesis of Thyrotropin'7th ED. Philadelphia .Lippinscott- Raven,190-207.

[31]. Xess, A.; Gupta, A.; Kumar, U.; Sharma, H.P. and Prasad, K.M. (1999): Evaluation of thyroid hormones in chronic renal failure.Indian.J.Pathol.Microbial,42(2):129-133.

[32]. Yilmaz, M.E and Kiraz, M. (2000): The Evaluation of serum Zinc and Copper Levels in Hemodialysis Patients in southeast Turkey.Dialysis and Transplantation, 29(11):718-720.

[33]. Zadrazil , J. (2011): Aetiology and a clinical picture of chronic renalfailure: 607-613.

[34]. Zeraati, A.A.; Layegh, P.; Famili, Y.; Naghibi, M.; Sharifipour,F.and Sarabi, Z.S. (2011): Serum Triiodothyronine Level as an indicator of Inflammation in Patients Undergoin Dialysis. Iranian journal of Kidney Diseases, $5: 38-40$.

[35]. Zoccali,c.; Tripepi,G.;Cutrupi,S.;Pizzini,P. and Mallamaci,F. (2005): A new facet of inflammation in end stage renal disease.J.AM.Soc.Nephrol.16:2789-2795. 